\title{
The Application of Wireless Broadband Communication for Smart Power Distribution and Utilization Networks
}

\author{
FENG Yongjun ${ }^{1,}$, LI Mingxia ${ }^{2, b}$, LIU Xiaohua ${ }^{1, c}$, YUAN Mingfeng ${ }^{1, d}$ and HE \\ Yeshen ${ }^{3, e, *}$ \\ ${ }^{1}$ State Grid Xinjiang Electric Power Corporation Marketing Department, Xinjiang China \\ ${ }^{2}$ State Grid Xinjiang Electric Power Corporation Electric Power Research Institute, Xinjiang China \\ ${ }^{3}$ State Grid Information \& Telecommunication Group \\ afengyongjun6160@126.com.cn, b543569362@qq.com.cn, ${ }^{\mathrm{c}} 1173308892 @ q q . c o m,{ }^{\mathrm{d}} \mathrm{ymf12} @ 126 . c$ \\ om, ${ }^{e}$ nelsonhonger@189.cn*
}

Keywords: Wireless broadband communication, smart power distribution and utilization network, smart grid, application framework.

\begin{abstract}
The power wireless broadband communication is one of key support for smart power distribution and utilization networks, which is usually at the end of the power system communication network and deployed in where the optical fiber communication is not easy to achieve. In this paper, we design an application framework for the power wireless broadband communication. Meanwhile, we also present novel schemes of the application framework for the smart power wireless broadband communication including the technology scheme and access scheme. The operation shows our solution can effectively sense the environmental object and monitor the intelligent terminals in power distribution and utilization.
\end{abstract}

\section{Introduction}

The power wireless broadband communication is one of key support for smart power distribution and utilization networks, which is usually at the end of the power system communication network and deployed in where the optical fiber communication is not easy to achieve. The power wireless broadband communication has some good characteristics, such as large coverage area, the signal dispersion. It is a reasonable way to build a reliable, real-time, safe wireless communication network for electricity networks[1,2].

In the smart power wireless broadband communication network, there are some place are not suitable to deploy the optical fiber communication due to economic reasons or hard environments, for example, in the suburbs, the open countryside or in the basement of the complex ground conditions. Therefore, it is very necessary to employ the wireless broadband communication technologies to support the communication in the power distribution and utilization networks. Usually, according to the requirements of smart power distribution and utilization businesses, we divide the electric users into four types: category A: specifically large user, such as the large industrial factories; category B medium user, such as normally factories; category $\mathrm{C}$ : general industrial and commercial users; category D: single-phase general industrial and commercial users; category E and F usually are residential users assess public distribution transformer metering point as shown in Table 1[1].

Table 1. Throughput Requirement

\begin{tabular}{ccccccc}
\hline Communication & \multicolumn{7}{c}{ Model } & A & B & C & D & E & F \\
\cline { 2 - 7 } & 8328.6 & 8328.6 & 7540.9 & 3677.5 & 3677.5 & 7685.0 \\
\hline Whole Model & 8303.0 & 1803.0 & 1897.5 & 1133.6 & 1133.6 & 1517.1 \\
\hline Base Model & 1803.6
\end{tabular}


Moreover, the wireless broadband communication also deployed in power distribution automation for collecting information from the switching stations, substations, ring counter, pole-mounted transformer and switches and other equipment using DL /T6345-101 and a remote loop protocol [3].

In this paper, we design an application framework for the power wireless broadband communication including the terminal layer, the base station, the core network and the operation and maintenance center. Meanwhile, we also present novel schemes of the application framework for the smart power wireless broadband communication including the technology scheme and access scheme. The schemes can be used in the electricity information collection, distribution electrical automation, load management, and emergency communications and other smart grid services.

\section{Model}

In our solution, the application framework supports the whole application of smart power distribution and utilization communication system, which can be divided into parts as shown in fig .1.

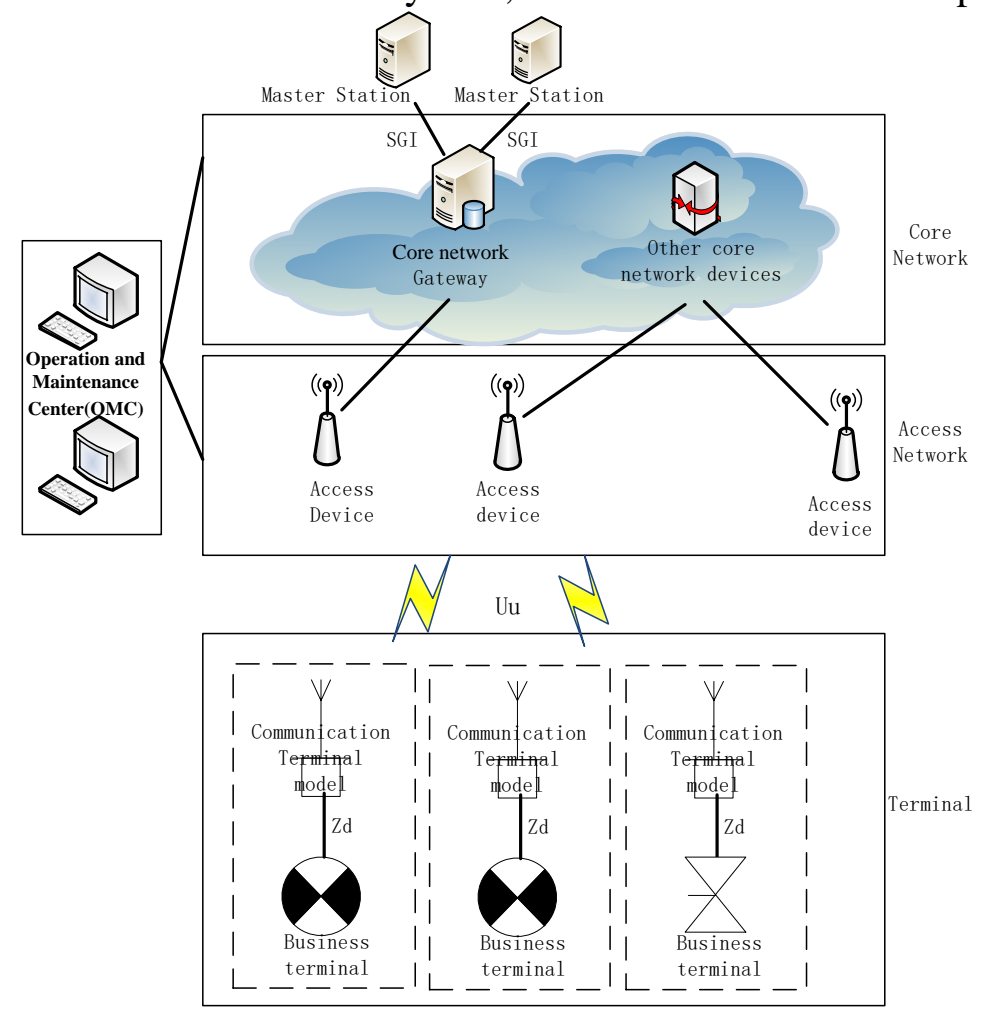

Fig.1 The power wireless broadband network system

The terminal devices. By the wireless communication, the terminal devices usually transfer the collected data form meters, concentrators, etc. to base station. These terminals have a lot of basic data which are need for data cleansing and integration, then transferred to core network by base station, and finally supplied to power business applications.

The base station. The access equipment is mainly responsible for the access function of the terminal device. The base station communicates with the terminal through the air interface, and is responsible for transmitting the radio signal, the reception and radio resource management. Meanwhile, it also connects to the access network and the core network through the S1 interface, the user is responsible for routing information to the core network, and encrypts the signal and data as well as Internet Protocol header compression.

The core network. The core network is the bridge between the main station and the access network communication. It connects to base station through the S1 interface and connect to the master station through SGi interface [4]. The main function of the core network include: signaling processing and signaling functions, such as non-access layer signaling and S1 signaling processing and transmission functions; mobility management functions, including registration, accessibility and connectivity management terminal etc; data processing functions, including hosting management and 
user plane data transfer capabilities, such as data processing for transmission SGi port for data processing and transmission base station; subscription data management functions.

Operation and Maintenance Center. The Operation and maintenance center supports functions as follow :the configuration management, fault management, performance management, logging, and security capabilities for network operation and maintenance, and improves the efficiency of the grid and reduces the network operating and maintenance costs.

\section{Technology Scheme}

Link budget. Considering the fact of the sparse base station deployments, we choose the Okumura-Hata computation model [6] which is suitable for large scale area (the cell radius greater than $1 \mathrm{~km})$. And the frequency range is $150 \mathrm{MHz} 1500 \mathrm{MHz}$.

The Empirical formula of Okumura-Hata is as $\mathrm{Eq}(1)$ :

$$
L_{m}=69.55+26.16 \log (f)-13.82 \log \left(h_{t e}\right)-a\left(h_{r e}\right)+\left[44.9-6.55 \log \left(h_{t e}\right)\right] \log (d)
$$

Where $f$ denotes the carrier frecuncy; $h_{t e}$ indicates the effective height of the transmitting antenna; and $h_{r e}$ denotes the receiving antenna effective height; $d$ is the distance between the transmitter and the receiver, $a\left(h_{r e}\right)$ is a mobile antenna correction factor, its value depending on the environment.

We just consider about the situation in urban and countryside, and then got the modified Okumura-Hata formulas as follow:

For Urban:

$$
L_{m}=L(\text { Urban })-2[\log (f / 28)]^{2}-5.4
$$

For countryside:

$$
L_{m}=L(\text { Countryside })-4.78[\log (f)]^{2}-18.33 \log (f)-40.98
$$

Usually, we can adjust the parameters for $\operatorname{Eq(2),~} \mathrm{Eq(3)}$ according to the topography when we deploy the base stations. Moreover, wae can adopt the COST-231 Hata model in the base station crowded area[6].

Wireless broadband technoloty. When we employ the $230 \mathrm{MHz}$ communiation for power wireless broadband communication[7], we define unified number for each resource unit (RB) allocation $25 \mathrm{kHz}$ and facilitate the system operator on the $230 \mathrm{MHz}$ frequency band. The downlink carrier deployment scheme is shown as fig 2.
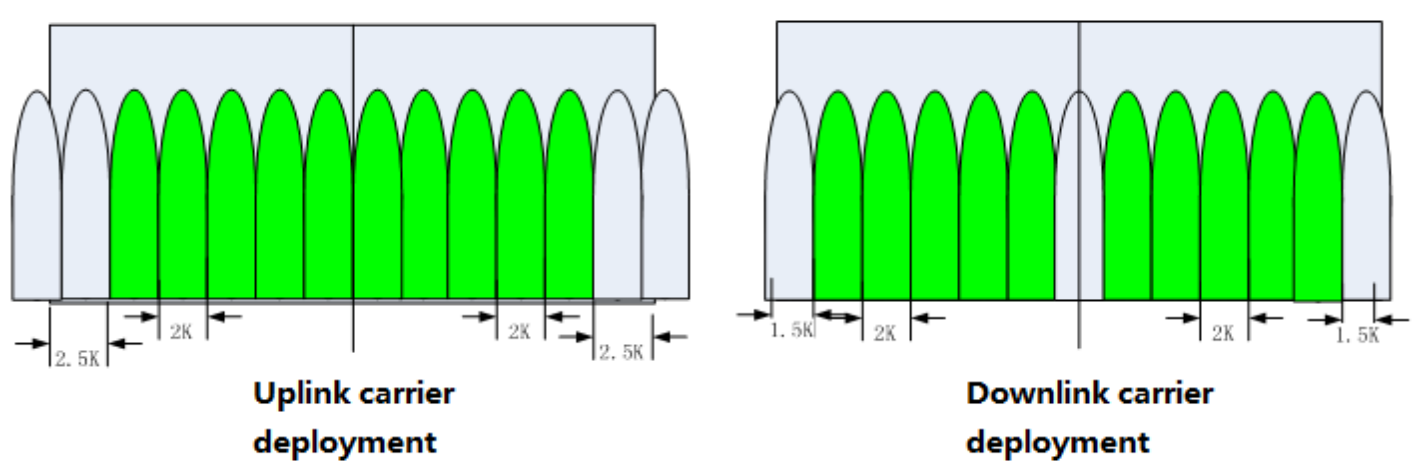

Fig 2. The carrier depolyment

\section{Access Scheme}

Broadband wireless access scheme. In the smart power wireless broadband communication, we adopt the OFDMA(Orthogonal Frequency Division Multiple Access) of TDD in the 230MHz. The OFDMA has some better characteristics than other normal FDMA, TDMA and CDMA, such as the high spectral efficiency, the ability of anti-frequency selective fading, and the low complexity 
receiver by the frequency domain equalization. OFDMA has good performance in the frequency domain resources and user partition, it can be flexible on the allocation of resources in discrete spectrum, ideal for use with carrier aggregation technology. Meanwhile, it can also be considered in conjunction with TDMA technology to get better results.

TDD duplex mode. In the duplex mode on mobile communication, there are mainly divided into FDD and TDD, and TDD is relatively greater flexibility than FDD. In the communication system of discrete narrowband spectrum, the distribution and the number of discrete channels are difficult to predict. The $230 \mathrm{MHz}$ system is also very difficult to predict. FDD mode requires advance for uplink and downlink frequency setting, which will inevitably lead the system flexibility decreases. However, the TDD can control the uplink and downlink channel capacity by time distinguish ratio, even if the frequency change, the uplink and downlink capacity is also changed, the ratio remains constant. In addition to this advantage, TDD transceiver design as well as less complex, lower cost advantages of the transceiver. Demodulation and modulation and coding selection process, we can use the same frequency uplink and downlink channel reciprocity characteristics, and reduce the feedback uplink and downlink channel quality.

\section{Summary}

In this paper, we present novel technology scheme and access scheme for the application of broadband wireless communication for the smart power distribution and utilization network. The schemes can be used in the electricity information collection, distribution electrical automation, load management, and emergency communications and other smart grid services. The laboratory experiments and operation show that our solution uses TD-LTE technology power private wireless communications network based on the power spectrum resources. It is a good solution for the large-scale, multi-measure communication in the smart grid.

\section{References}

[1] J.P Cao, J.M Liu, X.Z Li,, A Power Wireless Broadband Technology Scheme for Smart Power Distribution and Utilization Networks, Automation of Electric Power Systems, 2013,Vol(37)

[2] M.DAOUD, X. FERNANDO, On the communication requirements for the smart grid, Energy and Power Engineering, 2011,3(1):53-60.

[3] Ahmed, M., Ahmad, I., \& Habibi, D. (2015). Green wireless-optical broadband access network: energy and quality-of-service considerations.Optical Communications \& Networking IEEE/OSA Journal of, 7(7), 669-680.

[4] Mao, R., \& Julka, V. (2011). Wireless Broadband Architecture Supporting Advanced Metering Infrastructure. Vehicular Technology Conference (VTC Spring), 2011 IEEE 73rd (pp.1-13). IEEE.

[5] Chen, Y., Hwang, J. K., \& Wu, S. M. A reliable power line carrier and wireless data concentrator for broadband energy information network. IEEE Transactions on Consumer Electronics, 2003,49(4), 1054-1060.

[6] Singh Y. Comparison of Okumura-Hata and COST-231 Models on the Basis of Path Loss and Signal Strength. International Journal of Computer Applications, 2012, 59(11):37-41.

[7] Liu Y, Zhu E G, Liu X, et al. 230MHz Wireless Communication Network Architecture and Design of Power Consumption Information Collection System Based on WiMAX. Applied Mechanics \& Materials, 2014, 577:835-840.. 